\title{
Comfort with Motherhood in Late Pregnancy Facilitates Maternal Role Attainment in Early Postpartum
}

\author{
Yasuka Nakamura, ${ }^{1}$ Yoko Takeishi, ${ }^{2}$ Naoko Ito, ${ }^{2}$ Mizuki Ito, ${ }^{3}$ Fumi Atogami ${ }^{1}$ and \\ Toyoko Yoshizawa ${ }^{1}$
}

${ }^{1}$ Women's Health Nursing, Tohoku University Graduate School of Medicine, Sendai, Miyagi, Japan

${ }^{2}$ Tohoku University Hospital, Sendai, Miyagi, Japan

${ }^{3}$ Sendai Red Cross Hospital, Sendai, Miyagi, Japan

Quality of life, comfort, and wellbeing during pregnancy are essential for every country in the world. Pregnancy is considered a preparation period for becoming a mother. Maternal role development, including confidence and satisfaction as a mother, is important in the transition to motherhood. Negative psychosocial affect, such as increased anxiety and distress, during pregnancy adversely influences the childbirth experience and childcare, which contributes to postpartum depression. However, the impact of positive feelings on the maternal role development remains unclear. Therefore, the study purpose was to clarify the relationship between comfort in late pregnancy and maternal role attainment and childcare during early postpartum. We designed a descriptive, longitudinal, correlational study by using the Prenatal Comfort Scale, the Postpartum Maternal Role Confidence Scale, and the Postpartum Maternal Satisfaction Scale. Among 339 participants who had received care at a university hospital located in Sendai city in Japan, 215 subjects completed the longitudinal study by answering a questionnaire for the respective Scale late in their pregnancy or during early postpartum. The subjects consisted of 114 primipara (32.0 \pm 5.4 years) and 101 multipara (33.4 \pm 4.9 years). In primipara, comfort with motherhood was significantly correlated with maternal confidence regarding knowledge and childcare skills and maternal satisfaction. In multipara, comfort in late pregnancy was related to maternal confidence and satisfaction. Positive affect was related to maternal confidence and maternal satisfaction in early postpartum. Therefore, a prenatal nursing intervention helps women become more comfortable with impending motherhood, thereby promoting maternal role attainment after delivery.

Keywords: comfort; maternal confidence; maternal satisfaction; multipara; primipara

Tohoku J. Exp. Med., 2015 January, 235 (1), 53-59. C 2015 Tohoku University Medical Press

\section{Introduction}

Quality of life (QOL), comfort, and wellbeing during pregnancy have all been increasingly important in recent research. In Japan, the goals of the National Plan for the health of mothers and children 2001 to 2014, "Healthy Parents and Children 21," included ensuring a safe and comfortable pregnancy and childbirth, and providing assistance for infertility (Ministry of Health, Labour and Welfare 2010). The National Plan proposed that enhanced positive states, such as QOL, comfort, and wellbeing were required for pregnant women.

Pregnancy has been referred to as a preparation period for becoming a mother psychosocially, which includes the acceptance of the baby into one's self-system and living space (Rubin 1984). Shindo and Wada (1992) also defined pregnancy as a preliminary stage of the maternal role attain- ment process. Women prepare for motherhood during this period by creating a self-image as a mother, obtaining knowledge of the maternal role, and acquiring skills required for parenting (Shindo and Wada 1992). Klaus and Kennel (1982) also pointed out that important events for formulating the mother-infant attachment occur during pregnancy, delivery, and early postpartum; such events include pregnancy acceptance, fetal movement, and the perception of the fetus as a person, the delivery, and the child for the first time.

The associations between women's psychosocial status (e.g., incidence of depression or anxiety) during pregnancy and postpartum depression and child outcomes have been studied. For example, Faisal-Cury and Menezes (2012) studied risk factors for postnatal depression in 831 pregnant women. Results indicated that nearly $50 \%$ of postnatal women who had depressive symptoms had already shown

Received September 26, 2014; revised and accepted December 25, 2014. Published online Janaury 16, 2015; doi: 10.1620/tjem.235.53.

Correspondence: Yasuka Nakamura, Women's Health Nursing, Tohoku University Graduate School of Medicine, 2-1 Seiryo-machi, Aoba-ku, Sendai, Miyagi 980-8575, Japan.

e-mail: nakamurayasuka@ nursing.med.tohoku.ac.jp 
depressive symptoms during pregnancy, and women who had antenatal depression were 2.4 times more likely to present with postnatal depression compared to women who did not have such symptoms during pregnancy; thus, they concluded that antenatal depression strongly predicts postnatal depression. A large prospective study in Australia reported that antenatal depression, together with a prior history of depression and a low level of partner support, was the strongest independent antenatal predictor of postnatal depression (Milgrom et al. 2008). Edwards et al. (2008) also reported that women who had antenatal depression were significantly more likely to be depressed in the postnatal period. In Japan, Toukairin et al. (2010) studied risk factors for postnatal depression. It was suggested that pregnant women with economic anxiety who have difficulty consulting their husbands during the prenatal period are at high risk for postpartum depression. Correia and Linhares (2007) reviewed maternal anxiety in the prenatal period, and pointed out that high levels of maternal anxiety in the prenatal phase were associated with obstetric problems, emotional damages to fetal development, and behavioral problems in childhood and adolescence. Influences on child outcomes were also reported by other researchers. Luoma et al. (2001) reported that maternal prenatal depressive symptoms significantly predicted problematic behavior in children 8 and 9 years of age, and wellbeing in adolescent boys (Korhonen et al. 2012). However, it remains to be explored whether positive psychosocial status, defined as comfort, happiness, and enjoyment during pregnancy through the postpartum period, impacts childcare behavior and child outcomes.

Maternal role development is an essential component of the transition to motherhood and childrearing. Several studies have examined the relationship of maternal attachment during the prenatal period as a preparation period of motherhood on the relationship within the postnatal period. Women's fetal attachment was significantly related to infant attachment (Sato 2004) and postnatal attachment after 3 months and 1 year (Ohmura and Mitsuoka 2006). Sato (2005) reported that maternal attachment in the pregnancy period that was still present at one month influenced childrearing behavior at 18 months. However, there is little research focusing on the correlation between psychosocial states, especially positive states, during pregnancy and maternal role attainment after having a baby.

The presence of certain factors during pregnancy can increase the risk of child abuse; namely, a high risk of child abuse is associated with unintended pregnancy, minimal communication with family, including the husband, and having a spouse who does not contribute to childcare (Higuchi et al. 2004; Goto et al. 2005). Thus, it is important to determine the impact of maternal role attainment and comfort during pregnancy on subsequent childrearing behavior.

The purpose of this study was to examine the relationship between comfort in late pregnancy and maternal role attainment in early postpartum.

\section{Methods}

Patients and procedure

A descriptive correlational design with convenience sampling was used. A subset of data was used from a longitudinal study that examined changes in comfort during pregnancy and their influence on childbirth satisfaction and parenting in early postpartum (Nakamura 2012; Takeishi 2012). In this study, data were collected from participants in the 3rd trimester of their pregnancy and one month after delivery at one university hospital that provided prenatal visit and delivery support from January 2011 to March 2012. Recruitment posters were placed in an outpatient waiting space, and women who would be receiving continual maternal care after one month and were able to read Japanese received a questionnaire from the midwife. If they agreed to participate, they completed the anonymous questionnaire and sealed it for return in a ward collection box or by mail. Despite the longitudinal design, anonymization in a linkable fashion was taken. Each participant was managed by a unique ID number in this study. This study was approved by the Ethics Committee of Tohoku University Graduate School of Medicine (Approval number: 2010-429, 2012-1-195).

\section{Measurements}

Prenatal Comfort Scale: The Prenatal Comfort Scale (PCS), which measures positive affect in pregnant women, contains 35 items divided into five subscales (Takeishi et al. 2011). The five subscales are as follows: deepening relationships with husband growing to father [HUSBAND], interactions by moving of fetus [FETUS], support from communications with the people around [PEOPLE], realization of becoming a mother and attachment to the baby [MOTHER], and changes in myself during the pregnancy life [MYSELF]. Each item is rated on a 6-point Likert scale ranging from 1 (have not experienced) to 6 (experienced very much). The total score can range from 35-210, with higher scores indicating greater experiences of comfort. Concurrent validities of correlation with the Visual Analog Scale and the Pregnancy acceptance subscale of the Japanese version of Prenatal Self-Evaluation Questionnaire were 0.57 and 0.61 , respectively. Convergent and discriminant validities were ensured and the scaling success rate was $99.4 \%$ (Takeishi et al. 2011). Alpha internal consistency reliability of all items was 0.95 , and ranged from 0.81 to 0.92 for the five subscales (Takeishi et al. 2011). The internal consistency for this study was 0.97 for all items, and $0.88-0.95$ for the five subscales.

Postpartum Maternal Role Confidence and Maternal Satisfaction Scales: The Postpartum Maternal Role Confidence Scale (PMCS) and the Postpartum Maternal Satisfaction Scale (PMSS) were designed to measure perceived maternal role attainment during early postpartum. The PMCS contains 20 items categorized into the following 4 subscales: "Confidence of knowledge and skills for child care" [SKILL], "Ability to read infant cues" [CUES], "Ability to respond to infant's needs" [NEEDS], and "Establishment of how to fit into the role of "me and my baby" [FIT]. The PMSS consists of 9 items divided into 2 subscales: "Self-affirmation as a mother" [AFFIRM] and "Enjoyment gained from interacting with the infant" [ENJOY]. Concurrent validity was supported, as the scales were positively correlated with the self-esteem scale, and negatively correlated with a scale that measures anxiety about childrearing. Construct 
validity was indicated by the correlation between the scales, which ranged from 0.51 to 0.59 ; and the correlation between the subscales, which ranged from 0.34 to 0.72 (Maehara and Mori 2005). Cronbach's alpha was $0.89-0.88$, and $0.80-0.81$, respectively (Maehara and Mori 2005). The internal consistency of the PMCS and PMSS for this study was 0.91 and 0.91 for all items, and 0.74-0.85 and $0.78-0.79$ for the subscales.

Socio-demographic and obstetrical characteristics: Data on maternal age; partner's age; marital status; family composition; employment; gestational week; Japanese version of the Edinburgh Postnatal Depression Scale (EPDS) (Cox et al. 1987; Okano et al. 1996) at one month postpartum, which has been used as the screening test of postpartum depression internationally (Cox and Holden 1994); and obstetric history were obtained from the questionnaire. Data pertaining to the babies, such as the infant's weight and treatment for infant, were obtained from medical records.

\section{Statistical analyses}

SPSS version 19.0J was used for data analysis. Sociodemographic and clinical data were compared between primipara and multipara. Descriptive statistics were used to provide demographic characteristics and study variance. Chi-square tests, t-tests, MannWhitney tests, and analysis of variance were used for comparisons between two groups. Pearson's correlation analysis was conducted to assess the relationship among the study variables. The level of significance was $p<0.05$.

\section{Results}

\section{Samples}

The questionnaire was distributed to 379 women, and 339 women responded at least once during the longitudinal research period. In this study, we analyzed data from 215 women who answered the questionnaire both late in their pregnancy and during early postpartum. The socio-demographic and obstetrical characteristics data of the participants are presented in Table 1. Primipara women $(n=114)$ ranged from 17 to 44 years old; their mean age was 32.0 (s.D. $=5.4)$. Multipara women $(n=101)$ ranged from 18 to 44 years old; their mean age was 33.4 (s.D. $=4.9$ ). Husband's mean age across both groups was 33.6 (s.D. = 5.9 ) years old and 34.6 (s.D. $=5.8$ ) years old, respectively.
All women were married or had a partner. The cut-off point of EPDS was 8/9, per the recommendation of the survey for a Japanese population (Okano et al. 1996). There were no statistical differences between primipara and multipara women in socio-demographic or clinical variables, such as family composition, EPDS at one month postpartum, baby's weight, and treatment of infant.

Because comfort during pregnancy significantly differed between multipara and primipara, data from these two groups were analyzed separately.

\section{The PCS, PMCS, and PMSS scores}

These scores are shown in Table 2. Total score and scores for all subscales of the PCS except [MOTHER] were significantly higher in primipara than in multipara women $(p=0.030-<0.001)$. Conversely, total score and scores for all subscales of the PMCS were significantly lower in primipara women compared to multipara women $(p=0.003$ $<0.001)$. The total PMSS scores and subscale scores did not significantly differ between primipara and multipara women $(p=0.579,0.333)$.

\section{Relationship between comfort in pregnancy and postpartum maternal role}

The relationships between comfort variables in pregnancy and postpartum maternal role are presented in Tables 3 and 4. In primipara, comfort about [MOTHER] during late pregnancy significantly correlated with [SKILL] in postpartum $(r=0.220, p<0.01)$. The PCS total score and scores for all subscales except [HUSBAND] significantly correlated with total and subscale scores of the PMSS; [ENJOY] and [AFFIRM] $(r=0.256-0.376, p<0.01-0.001)$.

In multipara, the PCS total score, [PEOPLE], and [MOTHER] significantly correlated with the PMCS total score and scores of all PMCS subscales $(r=0.217-0.456$, $p<0.05-0.001)$. [HUSBAND], [FETUS], and [MYSELF] significantly correlated with the PMCS total score of and limited PMCS subscales $(r=0.228-0.391, p<0.05-0.001)$. The PMSS total score and scores for its subscales ([ENJOY] and [AFFIRM]) were correlated with the total PCS score and all PCS subscale scores $(r=0.264-0.579$,

Table 1. Demographic data.

\begin{tabular}{lcrr}
\hline & Primipara $(n=114)$ & Multipara $(n=101)$ & $95 \%$ CI \\
\hline Age $(\mathrm{M} \pm$ s.D. $)$ & $32.0 \pm 5.4 \mathrm{yrs}$ & $33.4 \pm 4.9 \mathrm{yrs}$ & $-2.81, .028$ \\
Answer weeks in 3rd trimester $(\mathrm{M} \pm$ S.D. $)$ & $34.7 \pm 1.3 \mathrm{wks}$ & $34.6 \pm 1.4 \mathrm{wks}$ & $-.351, .403$ \\
Answer day in postpartum $(\mathrm{M} \pm$ S.D. $)$ & $28.0 \pm 8.8$ days & $29.6 \pm 8.8$ days & $-4.412,1.149$ \\
Husband's age $(\mathrm{M} \pm$ s.D.) & $33.6 \pm 5.9 \mathrm{yrs}$ & $34.6 \pm 5.8 \mathrm{yrs}$ & $-2.818, .661$ \\
Baby's weight $(\mathrm{M} \pm$ S.D. $)$ & $2,973.3 \pm 395.9 \mathrm{~g}$ & $3,015.5 \pm 468.7 \mathrm{~g}$ & $-159.79,75.43$ \\
EPDS $(<8 / 9=<)^{*}$ & $89.3 \% / 10.7 \%$ & $93.3 \% / 6.7 \%$ & - \\
Nuclear family/expanded family* & $86.7 \% / 13.3 \%$ & $76.1 \% / 23.9 \%$ & - \\
Treatment for infant; yes/no* & $28.1 \% / 71.9 \%$ & $31.3 \% / 67.7 \%$ & - \\
\hline
\end{tabular}

Analyzed by $t$-test. EPDS, family type, treatment for infant (marked *) were analyzed by chi-square test. $95 \%$ CI, $95 \%$ confidence intervals. 
Table 2. Score of PCS, PMCS, and PMSS.

\begin{tabular}{|c|c|c|c|c|c|}
\hline & \multicolumn{2}{|c|}{ Primipara $(n=114)$} & \multicolumn{2}{|c|}{ Multipara $(n=101)$} & \multirow{2}{*}{$p$-value } \\
\hline & Mean & S.D. & Mean & S.D. & \\
\hline [PCS-TOTAL] & 171.9 & 21.9 & 160.1 & 29.8 & $.002 * *$ \\
\hline [HUSBAND] & 40.7 & 6.2 & 36.8 & 9.1 & $.000 * * *$ \\
\hline [FETUS] & 35.9 & 5.1 & 33.6 & 6.1 & $.003 * *$ \\
\hline [PEOPLE] & 38.8 & 5.9 & 36.2 & 7.2 & $.004 * *$ \\
\hline [MOTHER] & 36.0 & 4.9 & 34.9 & 5.3 & .101 \\
\hline [MYSELF] & 20.0 & 4.7 & 18.5 & 5.3 & $.030 *$ \\
\hline [PMCS-TOTAL] & 54.5 & 10.3 & 62.8 & 10.6 & $.000 * * *$ \\
\hline [SKILL] & 7.9 & 2.4 & 9.7 & 2.1 & $.000^{* * *}$ \\
\hline [CUES] & 20.6 & 3.5 & 22.3 & 4.6 & $.003 * *$ \\
\hline [NEEDS] & 14.5 & 4.3 & 17.6 & 4.3 & $.000 * * *$ \\
\hline$[\mathrm{FIT}]$ & 11.6 & 2.6 & 13.1 & 2.3 & $.000 * * *$ \\
\hline [PMSS-TOTAL] & 30.5 & 4.9 & 31.0 & 4.5 & .400 \\
\hline [ENJOY] & 14.1 & 2.1 & 14.2 & 2.0 & .579 \\
\hline [AFFIRM] & 16.4 & 3.1 & 16.8 & 3.0 & .333 \\
\hline
\end{tabular}

PCS, Prenatal Comfort Scale; PMCS, Postpartum Maternal Role Confidence Scale; PMSS, Postpartum Maternal Satisfaction Scale; HUSBAND, deepening relationships with husband growing to father; FETUS, interactions by moving of fetus; PEOPLE support from communications with the people around; MOTHER, realization of becoming a mother and attachment to the baby; MYSELF, changes in myself during the pregnancy life; SKILL, confidence of knowledge and skills for child care; CUES, ability to read infant cues; NEEDS, ability to respond to infant's needs; FIT, establishment of how to fit into the role of 'me and my baby'; AFFIRM, self-affirmation as a mother; ENJOY, enjoyment gained from interacting with the infant.

$* p<.05, * * p<.01, * * * p<.001$.

Table 3. Relationship between comfort in pregnancy and postpartum maternal role (primipara; $n=114$ ).

\begin{tabular}{|c|c|c|c|c|c|c|c|c|}
\hline & $\begin{array}{l}\text { [PMCS- } \\
\text { TOTAL] }\end{array}$ & [SKILL] & [CUES] & [NEEDS] & {$[\mathrm{FIT}]$} & $\begin{array}{l}\text { [PMSS- } \\
\text { TOTAL] }\end{array}$ & [ENJOY] & [AFFIRM] \\
\hline [PCS-TOTAL] & & & & & & $.313 * *$ & $.296^{* *}$ & $.287 * *$ \\
\hline \multicolumn{9}{|l|}{ [HUSBAND] } \\
\hline [FETUS] & & & & & & $.265 * *$ & $.237 * *$ & $.256^{* *}$ \\
\hline [PEOPLE] & & & & & & $.278 * *$ & $.258 * *$ & $.261 * *$ \\
\hline [MOTHER] & & $.220 * *$ & & & & $.376^{* * *}$ & $.355^{* * *}$ & $.346^{* * *}$ \\
\hline [MYSELF] & & & & & & $.328 * *$ & $.278 * *$ & $.318^{* *}$ \\
\hline
\end{tabular}

PCS, Prenatal Comfort Scale; PMCS, Postpartum Maternal Role Confidence Scale; PMSS, Postpartum Maternal Satisfaction Scale; HUSBAND, deepening relationships with husband growing to father; FETUS, interactions by moving of fetus; PEOPLE support from communications with the people around; MOTHER, realization of becoming a mother and attachment to the baby; MYSELF, changes in myself during the pregnancy life; SKILL, confidence of knowledge and skills for child care; CUES, ability to read infant cues; NEEDS, ability to respond to infant's needs; FIT, establishment of how to fit into the role of 'me and my baby'; AFFIRM, self-affirmation as a mother; ENJOY, enjoyment gained from interacting with the infant.

$$
* p<.05, * * p<.01, * * * p<.001 \text {. }
$$

$p<0.001)$. Particularly strong positive correlations were observed between the PCS and PMSS total scores, [MOTHER] and the PMSS total score, and [MOTHER] and [ENJOY] $(r=0.533-0.579, p<0.001)$.

\section{Discussion}

The participants in this study were women who received continuous care from pregnancy through to beyond one month postpartum. The two groups compared, primip- ara and multipara, did not significantly differ in demographics, child outcome, or EPDS. This suggests that these two groups were homogenous with regard to demographics and the presence of negative psychological aspects, such as EPDS screening; thus, the two groups differed only in terms of parity. Given this, the relationship between comfort in late pregnancy and maternal confidence and satisfaction in early postpartum could be explored in terms of parity differences. 
Table 4. Relationship between comfort in pregnancy and postpartum maternal role (multipara; $n=101$ ).

\begin{tabular}{|c|c|c|c|c|c|c|c|c|}
\hline & $\begin{array}{l}\text { [PMCS- } \\
\text { TOTAL] }\end{array}$ & [SKILL] & [CUES] & [NEEDS] & {$[\mathrm{FIT}]$} & $\begin{array}{l}\text { [PMSS- } \\
\text { TOTAL] }\end{array}$ & [ENJOY] & [AFFIRM] \\
\hline [PCS-TOTAL] & $.403 * * *$ & $.283^{* *}$ & $.456^{* * *}$ & $.371 * * *$ & $.217^{*}$ & $.533 * * *$ & $.481 * * *$ & $.480 * * *$ \\
\hline [HUSBAND] & $.280 * *$ & & $.346^{* *}$ & $.271 * *$ & & $.395 * * *$ & $.374 * * *$ & $.343 * * *$ \\
\hline [FETUS] & $.370 * * *$ & $.228^{*}$ & $.466^{* * *}$ & $.313^{* *}$ & & $.499 * * *$ & $.481 * * *$ & $.430 * * *$ \\
\hline [PEOPLE] & $.440 * * *$ & $.288^{* *}$ & $.482^{* * *}$ & $.394 * * *$ & $.245^{* *}$ & $.499 * * *$ & $.400 * * *$ & $.487 * * *$ \\
\hline [MOTHER] & $.421 * * *$ & $.337 * *$ & $.408^{* * *}$ & $.404 * * *$ & $.280^{* *}$ & $.573^{* * *}$ & $.579^{* * *}$ & $.474 * * *$ \\
\hline [MYSELF] & $.311^{* *}$ & & $.391 * * *$ & $.282 * *$ & & $.395 * * *$ & $.264 * * *$ & $.420 * * *$ \\
\hline
\end{tabular}

PCS, Prenatal Comfort Scale; PMCS, Postpartum Maternal Role Confidence Scale; PMSS, Postpartum Maternal Satisfaction Scale; HUSBAND, deepening relationships with husband growing to father; FETUS, interactions by moving of fetus; PEOPLE support from communications with the people around; MOTHER, realization of becoming a mother and attachment to the baby; MYSELF, changes in myself during the pregnancy life; SKILL, confidence of knowledge and skills for child care; CUES, ability to read infant cues; NEEDS, ability to respond to infant's needs; FIT, establishment of how to fit into the role of 'me and my baby'; AFFIRM, self-affirmation as a mother; ENJOY, enjoyment gained from interacting with the infant.

$* p<.05, * * p<.01, * * * p<.001$.

In this study, the PCS total and subscale scores of firsttime mothers were significantly higher than those of multiparous women, but comfort with becoming a mother and attachment to the baby showed only non-significant trends. Takeishi (2009) reported that primipara presented significantly more comfortable experiences than multipara, except for comfort-related interactions resulting from fetal movement. The present findings differ from those of Takeishi's study, but the sample in Takeishi's study comprised not only pregnant women who were in the 3rd trimester, but also women in their 1 st and 2 nd trimesters. If the sample consisted only of women in their 3rd trimester, such differences may not have been observed.

Multipara women had significantly higher maternal role confidence than did primipara. It was described that maternal confidence is acquired through interaction with the baby and caring for the baby in early postpartum (Mercer and Ferketich 1995), and is related to parity (Maehara and Mori 2005; Liu et al. 2012). The present findings would reflect those of previous studies, as these previous results are understandable. On the other hand, maternal role satisfaction did not differ significantly between the two groups. A similar result was found by Maehara and Mori (2005), and they suggested that mothers could enjoy interacting with their infants and experience self-affirmation as a mother independent of childrearing skill level.

In primipara, an association between comfort in late pregnancy and maternal confidence in early postpartum was not observed, but these variables were significantly related to maternal satisfaction. This indicates that because maternal confidence includes the ability to properly perform childrearing tasks (Maehara and Mori 2005), it is likely acquired through actual child care. For promoting maternal confidence, it is better to provide nursing care, which bolsters maternal confidence through actual childrearing after birth than during pregnancy. On the other hand, there was a significant correlation between maternal satisfaction and comfort in late pregnancy, especially comfort about realizing to becoming a mother and attachment to the baby $(r=$ $0.346-0.376, p<0.001)$. A nursing intervention to promote comfort with becoming a mother and attachment to the baby would likely facilitate greater maternal satisfaction. Moreover, the correlation between maternal confidence and maternal satisfaction $(r=0.51-0.59, p<0.01)$ (Maehara and Mori 2005) and between comfort about [MOTHER] in late pregnancy and [SKILL], a PMCS subscale, suggest such an intervention might encourage overall maternal confidence.

In contrast to primipara, comfort in late pregnancy for multipara was broadly related to maternal confidence in early postpartum. In particular, comfort about relationships with husband growing to father in late pregnancy was associated with maternal satisfaction. In addition, during pregnancy, multipara were responsible for rearing elder children. It is presumed that if women are comfortable about becoming mothers during pregnancy, their childrearing situation will also be positive. Evidence suggests that mothers' anxiety and stress levels are low if they have a positive relationship and good communication with their spouse (Hashizume and Taniguchi 2008; Terada et al. 2013). Another study showed that mothers are more likely to feel positive if their husbands are supportive and recognize the difficulties inherent in child rearing (Nagata et al. 2012; Sato 2012; Fujioka et al. 2013). Multipara women who showed higher PCS scores might have had better relationships with their husbands. Further study is needed to assess the association between comfort during pregnancy and the couple's relationship. Potentially, an intervention that promotes a positive marital relationship might lead to a more comfortable pregnancy life and consequently improve maternal confidence. Maternal confidence is one of the components of affirmative feelings towards childrearing (Shimada et al. 2003), related to maternal resilience (Nagumo et al. 2013), and a factor in difficult feelings regarding child care (Ida 2013) in Japan. Thus, it is impor- 
tant to focus on maternal confidence in the postpartum period.

Overall, the present results indicate a relation between comfort in late pregnancy and maternal satisfaction in early postpartum. As this relationship has not been previously reported, it represents new knowledge for health care providers. We suggest that a comfortable experience during pregnancy might facilitate maternal satisfaction, which includes self-affirmation as a mother and enjoyment gained from interacting with the infant in early postpartum, and consequently increase emotional maternal wellbeing. The promotion of maternal role attainment should begin during pregnancy and continue throughout the transition to parenthood.

\section{Limitations}

This study was a part of a longitudinal research project conducted from participants' 1 st trimester of pregnancy to one month after birth. A total of 379 questionnaires were distributed, and 289 women responded in their 3rd trimester; thus, the participation rate was $74.4 \%$. A total of 215 women answered in their 3rd trimester and one month after delivery, rendering the dropout rate $25.6 \%$. Reasons for discontinuing participation included changing hospitals, intrauterine fetal death, fetal abnormalities, and forgetting to return materials. As the women became very busy during the perinatal period with tasks related to preparing for the baby, it was challenging for many of them to continue participating in the study. Another reason for attrition may have been the lack of incentive for completing the questionnaires. Therefore, it is difficult to claim with certainty that the participants of this study represent new mothers in general, so this should be considered when interpreting the present results. However, since rewards were not offered, the answers given may have been more reliable. The sample in this study was recruited from only one university hospital; thus, it is possible that the data do not reflect the characteristics of women with low-risk pregnancies, or who received care at a clinic or birth center. Consequently, there might be a significant difference between comfort in late pregnancy and maternal confidence in early postpartum. In addition, it was difficult to clarify the influence of the marital relationship on comfort in late pregnancy and maternal role attainment in early postpartum. Further studies should examine the role of the marital relationship and the family to adequately explore the influence of comfort on maternal confidence and satisfaction in women.

\section{Conclusion}

In this longitudinal study, which extended from pregnancy through to postpartum, we explored the association between comfort in late pregnancy and maternal role attainment in early postpartum. In primipara, a relationship between comfort during pregnancy and maternal confidence was not detected; however, comfort was related to maternal satisfaction. On the other hand, in multipara, comfort in late pregnancy was associated with maternal confidence and maternal satisfaction in early postpartum. Because there was a relationship between maternal role attainment and comfort associated with the husband in particular, a possible effect of the marital relationship has been suggested. A nursing intervention that encourages women's comfort during pregnancy will likely promote maternal role attainment after birth.

\section{Acknowledgments}

This work was supported by a MEXT (The Ministry of Education, Culture, Sports, Science and Technology) KAKENHI Grant Number 22792214, JSPS (Japan Society for the Promotion of Science) KAKENHI Grant Number 24593350. Part of this study was presented at the 32nd Academic Conference of Japan Academy of Nursing.

\section{Conflict of Interest}

The authors declare no conflict of interest.

\section{References}

Correia, L.L. \& Linhares, M.B. (2007) Maternal anxiety in the pre- and postnatal period: a literature review. Rev. Lat. Am. Enfermagem, 15, 677-683.

Cox, J.L. \& Holden, J.M. (1994) Perinatal Psychiatry: Use and Misuse of the Edinburgh Postnatal Depression Scale, Gaskell, London.

Cox, J.L., Holden, J.M. \& Sagovsky, R. (1987) Detection of postnatal depression. Development of the 10-item Edinburgh Postnatal Depression Scale. Br. J. Psychiatry, 150, 782-786.

Edwards, B., Galletly, C., Semmler-Booth, T. \& Dekker, G. (2008) Does antenatal screening for psychosocial risk factors predict postnatal depression? A follow-up study of 154 women in Adelaide, South Australia. Aust. NZ J. Psychiatry, 42, 51-55.

Faisal-Cury, A. \& Menezes, P.R. (2012) Antenatal depression strongly predicts postnatal depression in primary health care. Rev. Bras. Psiquiatr., 34, 446-450.

Fujioka, N., Kato, N. \& Hamada, N. (2013) The fact-finding concerning the difficulties of rearing of women with one-yearold children and the satisfaction with their husband's participation in childcare. Japanese Journal of Maternal Health, 54, 173-181.

Goto, A., Yasumura, S., Yabe, J., Anazawa, Y. \& Hashimoto, Y. (2005) Association of pregnancy intention with parenting difficulty in Fukushima, Japan. J. Epidemiol., 15, 244-246.

Hashizume, M. \& Taniguchi, A. (2008) Child-rearing anxiety of mothers working as a nurse focusing on marital communications. Naganoken Kango Kenkyuu Gakkai Ronbunshu, 28, 34-36.

Higuchi, H., Tsubokawa, T., Takahashi, Y., Utakawa, T., Shirakawa, N. \& Yamada, K. (2004) High-risk factors of child abuse through childcare survey for preventing child abuse. The Japanese Journal for Public Health Nurse, 60, 1006-1013.

Ida, A. (2013) Concept analysis of "mother's child care diffuculty feeling" in Japan using Rogers's methods of concept analysis. Journal of Japanese Society of Human Caring Research, 4, 23-30.

Klaus, M.H. \& Kennel, J.H. (1982) Parent-Infant Bonding, 2nd ed., Mosby Company, Missouri, CV.

Korhonen, M., Luoma, I., Salmelin, R. \& Tamminen, T. (2012) A longitudinal study of maternal prenatal, postnatal and concurrent depressive symptoms and adolescent well-being. $J$. Affect. Disord., 136, 680-692.

Liu, C.C., Chen, Y.C., Yeh, Y.P. \& Hsieh, Y.S. (2012) Effects of maternal confidence and competence on maternal parenting 
stress in newborn care. J. Adv. Nurs., 68, 908-918.

Luoma, I., Tamminen, T., Kaukonen, P., Laippala, P., Puura, K., Salmelin, R. \& Almqvist, F. (2001) Longitudinal study of maternal depressive symptoms and child well-being. $\mathrm{J}$. Am. Acad. Child Adolesc. Psychiatry, 40, 1367-1374.

Maehara, K. \& Mori, E. (2005) Development of the postpartum maternal confidence scale and the postpartum maternal satisfaction scale: reliability and validity. Journal of Chiba Academy of Nursing Science, 27, 9-18.

Mercer, R.T. \& Ferketich, S.L. (1995) Experienced and inexperienced mothers' maternal competence during infancy. Res. Nurs. Health, 18, 333-343.

Milgrom, J., Gemmill, A.W., Bilszta, J.L., Hayes, B., Barnett, B., Brooks, J., Ericksen, J., Ellwood, D. \& Buist, A. (2008) Antenatal risk factors for postnatal depression: a large prospective study. J. Affect. Disord., 108, 147-157.

Ministry of Health, Labour and Welfare (2010) Second Evaluation Report "Healthy Parents and Children 21". http://www.mhlw.go.jp/shingi/2010/03/dl/s0331-13a015.pdf [Accessed: December 10, 2013].

Nagata, M., Nakamichi, Y., Noguchi, Y. \& Hirata, N. (2012) The factors which influence mothers' affirmative feelings towards childrearing at one and four months after giving birth. Japanese Journal of Maternal Health, 53, 329-336.

Nagumo, F., Murai, F. \& Emori, Y. (2013) Factors associated with confidence in childcare among mothers with low birth weight infants: an analysis with a focus on resilience. The Journal of Child Health, 72, 500-507.

Nakamura, Y. (2012) The relationships among comfort in pregnancy, birth satisfaction, and childrearing behavior, 20102011. In Report of Grant-in-Aid for Scientific Research (KAKENHI) for Young Scientists (B).

Ohmura, N. \& Mitsuoka, S. (2006) Prenatal and postnatal attachment of mother to her child and factors affecting longitudinal change of the attachment. The Journal of Child Health, 65, 733-739.

Okano, T., Murata, M., Masuji, F., Tamaki, R., Nomura, J., Miyaoka, H. \& Kitamura, T. (1996) Validation and reliability of Japanese version of EPDS (Edinburgh Postnatal Depression
Scale). Archives of Psychiatric Diagnostics and Clinical Evaluation, 7, 525-533.

Rubin, R. (1984) Maternal Identity and the Maternal Experience, Springer, New York, NY.

Sato, S. (2004) The effect of the attachment to pregnant women's fetus on the attachment to their infant. Jpn. Acad. Nurs. Sci., 24, 72-80.

Sato, S. (2005) The effect of maternal attachment on childcare attitude of mother at the birth post-18 month. The Journal of Child Health, 64, 507-514.

Sato, S. (2012) Study on the relationship of various factors comprising a sense of satisfaction from child rearing and assessment of the marital relationship as pertains to primiparas: focusing on the nuclear family during the initial stage of childrearing. Journal of Japan Academy of Midwifery, 26, 222-231.

Shimada, M., Emizu, F., Nagaoka, Y., Takahashi, H., Mori, T. \& Endoh, Y. (2003) Revision of a scale measuring puerpera's positive feeling toward child-care life. Journal of Japan Academy of Midwifery, 16, 34-45.

Shindo, S. \& Wada, S. (1992) Maternal Psychosocial Aspect and Nursing Care, Igakushoin, Tokyo.

Takeishi, Y. (2009) The development of prenatal comfort scale. Master of Nurse Science, Tohoku University, Miyagi.

Takeishi, Y. (2012) The Change in Comfort Through Pregnancy Progress, 2010-2011. In Reports of Grants-in-Aid for Scientific Research (KAKENHI) for Young Scientists (Start-up).

Takeishi, Y., Nakamura, Y., Atogami, F. \& Yoshizawa, T. (2011) Development of the prenatal comfort scale. Journal of Japan Maternity Nursing, 11, 11-18.

Terada, Y., Ohta, N. \& Matsuoka, M. (2013) Literature review about the childcare support during the childcare period. Shizuoka Journal of Maternal Health, 3, 49-58.

Toukairin, M., Onuma, E., Settai, M., Sugai, K., Matsuda, M. \& Akama, A. (2010) Verification of high-risk factors of postpartum depression from the background of the mother during pregnancy. Proceedings of The Japan Society of Nursing: Maternal Nursing, 40, 9-11. 